\title{
REVIEWS
}

\section{United We Fall}

\section{Arie Rip}

Michiel Schwarz and Michael Thompson, Divided We Stand: Redefining Politics, Technology and Social Choice (Hemel Hempstead, Herts. \& New York: Harvester Wheatsheaf, 1990), 176pp., $£ 35.00, £ 12.95$ pbk. ISBN 07450-04164 (-17872 pbk).

The title of this book turns out to reflect the complexity of its argument: Divided We Stand, at first reading, suggests an analysis of the conflicting viewpoints on technologies and desirable futures, and this is, indeed, what we get, even if refracted through the lens of cultural theory. 'Cultural theory' here is the Mary-Douglas-inspired analysis of differences in institutionally embedded cultures with the help of a two-by-two matrix of positions, depending on the strength of insideroutsider boundaries (the 'group' dimension) and the strength of role differentiation (the 'grid' dimension). For the basics of this approach the book refers to existing literature and forthcoming companion volumes. ${ }^{1}$

But there is more. On page 13, the full statement turns out to be: Divided we stand; united we fall. The normative side to the argument now becomes clear: we have to recognize the essential plurality of embedded cultures in order to avoid falling down. The conviction of the authors that they have found the key to viable social and technical development in modern society carries them through a variety of anecdotal examples and criticisms of other approaches, and culminates in a plea for plural assessments of technology, each time when conflicting certainties appear. Their concern about avoidable impasses and dead ends is one we can all share. The difficulty, of course, is to show how and why the recognition of plurality will actually lead to better outcomes. Here, the catchy phrases may promise more than can be delivered. 
Cultural theory has become fairly well known by now, and I will not bore the reader with an exposition of the individualists/ entrepeneurs, the hierarchists, the collectivists (called 'sectists' by others) and the fatalists (called 'ineffectuals' by others) and their respective styles or 'biases'. Schwarz and Thompson are clearly less partial in their labelling than Douglas and Wildavsky are, ${ }^{2}$ and in fact, try to argue for the value of the contribution of the collectivists (in their opening story about lavatory blocks being much improved thanks to the opposition of the German Greens to an earlier version of the blocks) and the non-negligible role of the fatalists (if only as the necessary backdrop to the controversies played out by the other three). The existence of the 'divisions' is necessary, they claim, and the aim should be mutual recognition rather than effacing or suppressing the differences. In other words:

[the] pluralism ... is essential, in the sense that each cultural bias - towards market solutions, towards hierarchical solutions, towards egalitarian solutions, and towards fatalistic acceptance - is not viable on its own. Individualists, for instance, need the hierarchists to enforce the law of contract, the hierarchists need the fatalists to sit on top of, the egalitarians need the inegalitarian excesses of the individualists and the hierarchists to criticize, and so on. (142)

The mutual dependencies sketched here are heterogeneous, to say the least. In fact, the authors are better at showing how things go wrong, or at least diverge, because of the working of the plural cultures, than they are at telling us how things go better once their existence is recognized and, presumably, acted upon.

Their fall-back line is that professions like engineering and the law, and other mediating institutions, because of their practical handling of situations, have accumulated and internalized 'tacit wisdom' about plural cultures. ' $[\mathrm{T}] \mathrm{hen}$ it is only a matter of designing the channels by which they can communicate that wisdom and thereby make it more explicit' (146). And if there is not enough of this tacit wisdom, '[m]ediating institutions ... should be designed (or redesigned) so that they always contain the requisite variety' (147). If readers are doubtful about the tacit wisdom of engineers in this respect, there is a further line of defence: the Dutch, because of their peculiar sociopolitical culture, have developed a particular form of technology assessment which tries to bring out plurality and make it useful for the assessments and choices that constitute technological developments. Being a Dutchman, and in addition strongly involved in the development of this so-called Constructive Technology Assessment, I 
can hardly defect. But it is a promise, rather than an achievement. As support for an ambitious claim about the need for plural rationalities, it falls short.

I am running ahead of the authors' argument here, and some of the earlier steps are much more convincing. The central insight, to my mind, is about patterns in uncertainty: 'Uncertainty, by definition, is unpredictable but reaction to uncertainty, though it can take a number of widely divergent forms, would appear to be strongly patterned - so predictable - as to be almost certain' (90). Such patterns can be found empirically, by tracing risk handling styles, problem definitions, 'myths of nature'. It is especially in controversies about technologies, about risks, about energy forecasts - in essence, about the question 'Whose, if anyone's future wins?' (25) - that the patterns can be characterized, and that the 'widely divergent forms' can be contained, so the authors assert, in a fourfold typology - that is, the four styles or cultural biases of cultural theory. There is, by now, enough case material (some of which is presented in the book) which indicates that the typology works - especially if handled flexibly. Case material also offers data about outcomes, but the interest of the authors is not so much in whose future wins, and why, but in 'where do the problem definitions come from?' (37). Thus they slant their analysis away from outcomes, and by going upstream rather than downstream, they create the hole in their argument that I noted above.

The strong patterning of reactions to uncertainty, and the cultural and institutional embedding of the patterns, is the stepping stone to a sustained and welcome criticism of the politics of interest explanations. In so far as politics of interest models consider interests as psychological facts, behaviour without any reference to social contexts (and the interactions going on, I add), the criticism is obvious. Schwarz and Thompson's additional point is that not all interests are possible, because actors must convincingly account for their actions in such a way as to be socially viable (52, emphasis added). Thus, 'political cultures' emerge and stabilize, and these should provide the framework for policy analysis, rather than given interests. Such a concept allows the analysis of the NIMBY (Not In My Back Yard) protest against the disposal of nuclear waste (which also allows an interest politics explanation) and protesting to prevent the disposal of nuclear waste in the middle of the Gobi desert (that is, NIABY: Not in Anyone's Backyard), in one framework (58-59). So far so good. One may be hesitant to accept 'political cultures' as a 
social phenomenon in its own right, but since specific patterns occur, one needs some cognitive or cultural structure as explanation.

Then there is a further shift, to the interesting question 'what leads one individual to support one policy ... and another individual to support another policy' (90), if we cannot interpret that in terms of interests or alignments only. This question leads the authors astray into a revival of the oversocialized conception of man, and into a bit of metaphysics.

Their first answer to the question is summarized as:

[T]hese declarations - these value-determined selections of fact $-\ldots$ are not so much conscious and voluntary decisions on the part of each policy actor as decisions that are supplied to him, in the form of unquestioned and self-evident assumptions, by the institutions in which he happens to be embedded. . . Cultural theory, in effect, deconstructs these largely unconscious and involuntary decision biases. (141)

Instead of the interest dope explanation, criticized in Chapter 4, we now appear to have the cultural-bias dope explanation! The difference, according to the authors, is that cultural theory has good reasons to posit its fourfold typology, while the interest explanation is always ad hoc. These good reasons, however, turn out, in the end, to be derived from a Platonist claim: ' . . the four myths of nature [one of the recurring examples of the nature and strength of the cultural biases] . . . continually resurface through the turbulent contingencies of history to reassert their essential timelessness' (85). And, more explicitly going 'from phenomena to the possibility of phenomena' (34):

these myths are eternal objects [,] not directly accessible. . . Eternal objects (like ways of worldmaking) have to do not with phenomena but with the possibility of phenomena, and these two levels are brought into relationship with one another by a third factor: the eternal object's mode of ingression into the actuality. This mode of ingression is accessible. It reveals itself to us in the form of recurrent identifiable elements (commitments, justifications, triumphs, tragedies, strategies, surprises, and so on) in our interactions with everything that is not us: our fellow humans and our circumambient cosmos. The deep reality that cultural theory enables us to get to grips with is our diverse involvements in the one world we all inhabit. (101)

Even if one doesn't mind the metaphysics, one should note the deep ambivalence in cultural theory that is contained in this position. In order to make the normative, and in a sense emancipatory point, about the need for the four cultures, the 'deconstruction with the help of cultural theory' must have effects; but at the same time, in the 
descriptive-analytical part of the argument, the bounds set by the four positions are emphasized. In other words, while we are all bound up in one or another of the four cultural biases, cultural theory, somehow, allows us to transcend them: "we should begin, coolly and dispassionately, by discovering what these different risk handling styles are' (120). The anthropologist can establish the legitimacy of the various rationalities, while actors are only concerned to impose their own rationality on others (paraphrased from 119; the sequel to this point will be taken up below).

In other places, the authors insist that one cannot get out of Plato's cave: there is no cosmic exile, no way of standing outside (110), or: since we are an essential part of the interactions, we can never stand right outside them (148). How can one ever escape? Michael Thompson has proposed, in earlier publications, that there is a fifth position in the grid-group scheme, that of the hermit, right in the middle, and unattached to grid or group. ${ }^{3}$ In the present book, it is the self-reliant mountaineer which is his ideal (and not unrelated to his personal experience). In places, the tacit wisdom of the engineer is also seen (as I noted already) as being able to handle the three active styles, without succumbing to any of them. Creating an additional, less dope-like position in the typology is one way to solve the ambivalence (provided one allows the hermit to come out of the mountains and preach). The other way is the Platonic route: being able to recognize eternal objects, seeing through the roman à clef (89), and thus transcending the bounds (the authors refer to A.N. Whitehead rather than Plato).

A third route should be considered, I think. In the same way that the reflexivity conundrums (not dissimilar from the present problematic) can be transcended by action, provided one remains accountable, we can say here that the cultural theorist, by becoming participant, can have effects, even though he will be forced to take up a position against, say, hierarchism, and thus play the role of the entrepreneurial individualist or the collectivist. Instead of dispassionateness, we would have constructive switching of positions. In fact, when one tries to reconstruct the authors' position on the basis of what they say in the book, one finds them switching positions. Although their own cognitive style, that of typology, appears to belong to the hierarchist position, the authors distantiate themselves from 'monster adjusting' (63), and from the pathology of the anticipatory mode, which implies, in E.M. Forster's words, 'staggering through life fully armed' (105), and go for some hierarchist bashing 
(105, 115-17 when arguing about irreducible uncertainty in the open systems we live in); in other places, they distantiate themselves from the individualist and especially the egalitarian viewpoints.

This third route is important for the argument of the book. The authors do discuss what they call 'stolen rhetoric' (73): actors taking up positions belonging to another bias, and doing so for strategic reasons (although the rhetoric may stick!). What they do not consider systematically is the constructive role of actors, or of participant analysts, in switching positions (provided they can be asked for their reasons, I would add). There are some intriguing excursions into dynamics already, for example on p. 100, where individualists are sketched as climbing on to the orthodox bandwagon (say, of the hierarchists in the 1950s and 1960s, or of the collectivists in the 1970s) as it gathers momentum, because they see a rich source of opportunities, and jumping off again as it begins to falter (for example, starting to exploit alternative energy and anti-pollution technology in the 1970s and 1980s). Thus, they impart a cyclical pattern to the totality - and, hopefully, an element of progress.

The thrust of the book, the authors insist, is that one should shift from the adversary to the exploratory mode. Not whose future will win, but how a better future can be reached. And all four cultural biases or rationalities have something to contribute. 'While the anarchic way that the rationalities have hitherto interacted can no doubt be improved upon, one thing is clear: diversity of conviction can produce major social benefits' (99, emphasis added). The continuing worry of the reader, of course, is whether the production of such benefits is historically contingent, or directly related to the existence and recognition of a diversity of conviction, and the particular diversity of the typology of cultural theory at that.

The exploratory mode is in order 'when we hear the ominous clash of contradictory certainties' (145), and we must then call for cultural analysis. But what should the cultural analyst (or the actor recognizing its insights) then $d o$ ? 'Intervene constructively', is the general idea (108). By being cool and dispassionate, and thus ineffectual? The authors are too brief on this point, as they are silent about the how and why of outcomes in general. Except, I should add, for some intriguing and memorable anecdotes, including the one about the senstive economist confronting the Great Energy Chief (of IIASA) with a plural rationality argument, and turning out to have been right (in a way), even if only after a lot of deconstructive work (including work by Michael Thompson). 
The general reader may well be interested and intrigued, and enjoy the parts of the book s/he can relate to. I am not a good judge here, being too much involved with these kinds of questions myself, as well as having had all sorts of discussions with the authors. ${ }^{4}$ The issues treated are certainly important. Instead of a juggernaut of technology, subjecting man and environment to its inexorable 'progress', we can now see that '[a]lmost all the technologies we now live with ( . . . ) went through their crucial early stages in a cloud of contradictory certainties (...)' (25). The authors continue and say that ' $[t]$ he challenge, therefore, is not to deny this cloud but to understand it'. Their positions are challenging, though not as challenging as the authors, complaining about the lack of acceptance of their theory (72), seem to think. While most of the examples are striking the real issue is to understand how and why we have got our present technology and how we can do better in the future. Here, the programmatic statements are in place, but I find the guidelines unconvincing, when they do no more than refer to the eternal interplay of cultural biases. We see the style of writing alternate between the felicitous phrase and plodding and somewhat repetitive argument. In general, the book attempts to combine Michiel Schwarz's ideas and earlier work about controversies and political cultures (as related to cultural theory), and technology assessment, with Michael Thompson's long- standing involvement with cultural theory, and his experiences in living among the energy tribes (and elsewhere). United, they fall short of being convincing. They need a better meta-cultural theory to make a stand.

- NOTES

1. M. Thompson, R. Ellis and A. Wildavsky, Cultural Theory (Boulder, CO: Westview Press, 1990).

2. M. Douglas and A. Wildavsky, Risk and Culture: an Essay on the Selection of Technical and Environmental Dangers (Berkeley, CA: University of California Press, 1985).

3. M. Thompson, 'A Three-Dimensional Model', and 'The Problem of the Centre: An Autonomous Cosmology', in M. Douglas (ed.). Essays in the Sociology of Perception (London: Routledge \& Kegan Paul, 1982), 31-63 and 302-38.

4. See, for example. Arie Rip, 'The Danger Culture of Industrial Society', in R. E. Kasperson and P. M. Stallen (eds), Communicating Risks to the Public (Dordrecht: Kluwer Academic Publishers, 1990), 347-67. 
Author's address Centre for Studies of Science, Technology and Society, University of Twente, PO Box 217, NL-7500 AE Enschede, The Netherlands.

Peter Galison, How Experiments End (Chicago, IL \& London: The University of Chicago Press, 1987), 288 pp., £31.95/\$39.95, £11.95/ \$15.95 pbk. ISBN 0-226-27914-6 (-27915-4 pbk).

Peter Galison's book contains an impressively detailed analysis of three episodes in the history of modern science. The first deals with a sequence of experiments on gyromagnetic effects - for example will the rotation of an iron bar cause it to become magnetized, and conversely will magnetization cause rotation? These experiments stem from Ampère's idea that magnetism is caused by tiny circulating electric currents, later identified with orbiting electrons. In 1915, Einstein worked on this subject. Galison's critical commentary on the design and conduct of these experiments shows the truly enviable level at which he is able to engage with the technical content of the work. He also brings out some interesting analogies between Einstein's experimental concerns and his earlier work in the Swiss federal patent office, when he was called upon to evaluate competing claims about magnetic compasses. The second case deals with the cosmic ray experiments leading to the discovery of an elementary particle, the mu-meson, in the 1930s. The third case concerns the discovery (in the early 1970s) again in elementary particle physics, of a decay process called 'weak neutral currents'. These studies sit between chapters of a more general character which are designed to set the work in the context of recent discussions in the philosophy and sociology of science.

Throughout the book a number of different aims are pursued in parallel. Partly the intention is to draw attention to the sheer importance of experiment itself. Along with a number of other

Social Studies of Science (SAGE, London, Newbury Park and New Delhi), Vol. 21 (1991), 186-89 